Revue internationale P.M.E.

Économie et gestion de la petite et moyenne entreprise

\title{
Innovations technologique et organisationnelle au sein de PME innovantes : complémentarité des processus, analyse comparative des mécanismes de diffusion
}

\section{Cécile Ayerbe}

Volume 19, numéro 1, 2006

URI : https://id.erudit.org/iderudit/1008488ar

DOI : https://doi.org/10.7202/1008488ar

Aller au sommaire du numéro

Éditeur(s)

Presses de l’Université du Québec

ISSN

0776-5436 (imprimé)

1918-9699 (numérique)

Découvrir la revue

\section{Citer cet article}

Ayerbe, C. (2006). Innovations technologique et organisationnelle au sein de PME innovantes : complémentarité des processus, analyse comparative des mécanismes de diffusion. Revue internationale P.M.E., 19(1), 9-34. https://doi.org/10.7202/1008488ar
Résumé de l'article

Les entreprises développant des produits ou procédés nouveaux sont également reconnues comme innovantes en matière d'organisation. La reconnaissance de l'innovation organisationnelle constitue une avancée significative pour l'analyse des processus d'innovation et permet de dépasser l'approche fragmentaire, centrée exclusivement sur la technologie, qui a longtemps dominé. Elle introduit toutefois un certain nombre d'interrogations. Parmi ces questionnements, la complémentarité entre les innovations technologique et organisationnelle ainsi que l'étude comparative des mécanismes de diffusion de ces innovations apparaissent encore peu explorées. Nous nous sommes donc intéressée à ces deux volets afin de rendre compte de la complexité de la dynamique d'innovation dans son ensemble. Plus précisément, notre article se propose de répondre aux questions suivantes :

- comment les innovations technologique et organisationnelle évoluent-elles et s'influencent-elles dans le temps?

- quels sont les mécanismes de diffusion de l'innovation à l'œuvre en matière de technologie et d'organisation?

Pour cela, nous avons eu recours à une analyse qualitative reposant sur une stratégie de cas multiples. Les PME innovantes ont été retenues comme terrain d'investigation. Les principaux résultats montrent l'existence de plusieurs phases, caractéristiques de l'évolution des différentes innovations réalisées par les PME sur longue période. Ils permettent en outre de relever les influences mutuelles entre innovations de différents types. De plus, l'étude des mécanismes de diffusion des innovations, visant aussi bien la technologie que l'organisation, autorise une compréhension plus fine de l'imbrication entre les différentes innovations réalisées par la PME et nous amène à repenser leurs liens en termes de « co-activation».
Ce document est protégé par la loi sur le droit d'auteur. L'utilisation des services d’Érudit (y compris la reproduction) est assujettie à sa politique d'utilisation que vous pouvez consulter en ligne.

https://apropos.erudit.org/fr/usagers/politique-dutilisation/ 


\title{
Innovations technologique et organisationnelle au sein de PME innovantes: complémentarité des processus, analyse comparative des mécanismes de diffusion
}

Cécile AYERBE GREDEG

Université de Nice Sophia-Antipolis

\section{MOTS CLÉS}

\section{Innovation technologique - Innovation organisationnelle Complémentarité - Mécanismes de diffusion PME innovantes - Étude qualitative}

\begin{abstract}
RÉSUMÉ
Les entreprises développant des produits ou procédés nouveaux sont également reconnues comme innovantes en matière d'organisation. La reconnaissance de l'innovation organisationnelle constitue une avancée significative pour l'analyse des processus d'innovation et permet de dépasser l'approche fragmentaire, centrée exclusivement sur la technologie, qui a longtemps dominé. Elle introduit toutefois un certain nombre d'interrogations. Parmi ces questionnements, la complémentarité entre les innovations technologique et organisationnelle ainsi que l'étude comparative des mécanismes de diffusion de ces innovations apparaissent encore peu
\end{abstract}

\section{L'AUTEURE}

CÉCILE AYERBE est maître de conférences à l'Université de Nice Sophia-Antipolis et membre du laboratoire CNRS «Groupe de recherche en droit, économie et gestion». Elle enseigne la stratégie et le management de l'innovation. Ses travaux portent principalement sur les liens entre les dimensions technologique et organisationnelle de l'innovation ainsi que sur la gestion de la propriété industrielle. Adresse: GREDEG, Université de Nice Sophia-Antipolis, 250, rue Albert-Einstein, bât. 2, 06560 Valbonne, France. Téléphone: 0493954395. Télécopieur: 04939543 22. Courriel <ayerbe@unice.fr>. 
explorées. Nous nous sommes donc intéressée à ces deux volets afin de rendre compte de la complexité de la dynamique d'innovation dans son ensemble. Plus précisément, notre article se propose de répondre aux questions suivantes:

- comment les innovations technologique et organisationnelle évoluent-elles et s'influencent-elles dans le temps?

- quels sont les mécanismes de diffusion de l'innovation à l'œuvre en matière de technologie et d'organisation?

Pour cela, nous avons eu recours à une analyse qualitative reposant sur une stratégie de cas multiples. Les PME innovantes ont été retenues comme terrain d'investigation. Les principaux résultats montrent l'existence de plusieurs phases, caractéristiques de l'évolution des différentes innovations réalisées par les PME sur longue période. Ils permettent en outre de relever les influences mutuelles entre innovations de différents types. De plus, l'étude des mécanismes de diffusion des innovations, visant aussi bien la technologie que l'organisation, autorise une compréhension plus fine de l'imbrication entre les différentes innovations réalisées par la PME et nous amène à repenser leurs liens en termes de «co-activation».

\begin{abstract}
Product and process innovative firms are also considered to be innovative in their organisational structure. The recognition of the major role of organisational innovation is a main contribution to the understanding of the whole innovation process. However it also raises a few questions such as the complementarity between technological and organisational innovations of the firm, or the comparative analysis of their diffusion mechanisms. These two main points have been investigated in order to outline the complexity of innovation process. Precisely, this article aims at answering the two following questions:

- how do technological and organisational innovations evolve and influence each other?

- what are their specific diffusion mechanisms?

To deal with these questions, we refer to a qualitative methodology based on multiple case studies of high technology based SMEs. The main results of this research stress three phases characteristic of the evolution of the various innovations within a longitudinal perspective. They also outline the mutual influence between innovations of different types. Moreover, the analysis of the diffusion mechanisms of technological and organisational innovations allows a precise understanding of their close relations within innovative SMEs. It also leads to reconsider the relations between technology and organisation in terms of "co-activation".
\end{abstract}

\title{
RESUMEN
}

A las empresas que desarrollan productos o procedimientos nuevos se las considera también como innovadoras en cuanto a la organización. El reconocimiento de la innovación organizacional constituye un avance significativo para el análisis de procesos de innovación y permite superar un planteamiento fragmentario centrado exclusivamente en la tecnología y que ha prevalecido desde hace mucho tiempo.

Revue internationale P.M.E., vol. 19, $\mathrm{n}^{\circ} 1,2006$

(C) 2006 - Presses de l'Université du Québec

Édifice Le Delta I, 2875, boul. Laurier, bureau 450, Québec, Québec G1V 2M2 • Tél.: (418) 657-4399 - www.puq.ca

Tiré de: Revue internationale P.M.E., vol. 19, $\mathrm{n}^{\circ}$ 1, sous la direction de Louis Raymond • PME1901N

Tous droits de reproduction, de traduction et d'adaptation réservés 
Sin embargo, conlleva algunos interrogantes como, por ejemplo, la complementaridad entre las innovaciones tecnológicas y organizacionales, así como el estudio comparativo de mecanismos de difusión de dichas innovaciones poco exploradas hasta la fecha. En nuestro artículo nos adentramos en estas dos fases con el fin de destacar la complejidad del proceso de la innovación en su conjunto. Así, pues en éste nos planteamos responder a las preguntas siguientes:

- ¿de qué modo las innovaciones tecnológica y organizacional van evolucionando con el tiempo y cómo se condicionan?

- ¿cuáles son los mecanismos de difusión de estas innovaciones?

Con este fin, recurrimos a un análisis cualitativo basado en una estrategia de varios casos. Las PyMEs innovadoras han sido nuestro campo de investigación. Los principales resultados ponen de relieve varias fases características de la evolución de las distintas innovaciones puestas en marcha por las PyMEs. Estos mismos nos permiten por otra parte especificar las influencias recíprocas entre las innovaciones de tipo diferente. Además, el estudio de los mecanismos de difusión de las innovaciones, más allá de las especificidades entre las tecnologías y organizaciones, facilita una comprensión más aguda acerca de la imbricación entre las diferentes innovaciones realizadas en una PyME y nos lleva a considerar los vínculos en terminos de "co-activación".

\section{ZUSAMMENFASSUNG}

Unternehmen, welche neue Produkte oder Verfahrensweisen entwickeln, werden gleichzeitig auch als innovativ in organisatorischen Fragestellungen betrachtet. Die Akzeptanz von organisatorischen Innovationen ist erheblicher Fortschritt für die Analyse der Innovationsprozesse. Sie erlaubt, die nur teilweise vorhandenen und nur auf technologische Fragestellungen basierenden Konzepte von früher zu überarbeiten und neu zu definieren. Die neue Betrachtungsweise impliziert aber einige wichtige Fragestellungen. Fragen zur Komplementarität von technologischen und organisatorischen Innovationen gehören dabei ebenso dazu, wie die Aufschlüsselung und Verbreitung der Mechanismen zwischen Innovationen. Aus der ganzen Komplexität der Fragestellungen rund um Innovationen haben wir folgende zwei Problemfelder genauer betrachtet und im vorliegenden Artikel dargestellt:

- Wie entwickeln sich die technologischen und organisatorischen Innovationen und wie beeinflussen sie sich gegenseitig im Prozess?

- Wie spielen die Mechanismen bei der Innovationsdiffusion hinsichtlich der Technologie und der Organisation?

Um diese beiden Fragen zu klären, haben wir eine qualitative Analyse bei mehreren innovativen KMU durchgeführt. Die Hauptergebnisse unterstreichen dabei die Existenz von mehreren Phasen, welche die Entwicklung von Innovationen über einen längeren Zeitraum bei kleinen und mittleren Unternehmen charakterisieren. Die Resultate zeigen, wie sich die verschiedenen Typen von Innovationen gegenseitig beeinflussen. Im Weiteren erlaubt die Studie, die Mechanismen der Innovationsdiffusion, jenseits der weitgehend bekannten Besonderheiten zwischen Innovationen in der Technologie und Organisation, genauer zu betrachten. Vor allem das gegenseitige Ineinandergreifen von verschiedenen Innovationen, im Sinne einer wechselseitigen "Co-Aktivierung" in KMU, kann neu reflektiert werden.

Revue internationale P.M.E., vol. 19, nº 1, 2006

(C) 2006 - Presses de l'Université du Québec

Édifice Le Delta I, 2875, boul. Laurier, bureau 450, Québec, Québec G1V 2M2 • Tél.: (418) 657-4399 - www.puq.ca

Tiré de: Revue internationale P.M.E., vol. 19, n 1, sous la direction de Louis Raymond • PME1901N

Tous droits de reproduction, de traduction et d'adaptation réservés 


\section{Introduction}

Les entreprises développant des produits ou procédés nouveaux sont également reconnues comme innovantes en matière d'organisation. Si de nombreuses recherches soulignent cette alliance entre innovations technologique et organisationnelle, peu d'auteurs ont cherché à mettre en évidence et à analyser ce phénomène. Dans ce contexte, notre recherche vise à explorer les complémentarités entre innovations technologique et organisationnelle au sein de PME technologiquement innovantes ${ }^{1}$. Elle s'inscrit ainsi dans le cadre des approches contemporaines de l'innovation qui reconnaissent l'importance de l'innovation organisationnelle comme vecteur d'innovation technologique (Ménard, 1994) et souhaitent mieux rendre compte des processus d'innovation au sein des PME (Chanal, 2002). Pour cela, nous avons choisi deux axes clés qui, en raison de l'état actuel des connaissances, nous semblent contribuer à l'amélioration de la compréhension des phénomènes d'innovation. Nous avons tout d'abord cherché à dépasser les approches dichotomiques qui ont longtemps marqué la littérature afin de tenir compte des liens de réciprocité entre les dimensions technologique et organisationnelle de l'innovation. Ce premier volet théorique a été complété par une analyse des mécanismes de diffusion des différentes innovations afin de souligner la complexité de la dynamique d'innovation dans son ensemble. Nous nous sommes volontairement limitée à la question de la diffusion, car ce champ d'investigation nous est apparu pertinent pour rendre compte de l'imbrication entre innovations technologique et organisationnelle. Cette recherche a donc pour but de répondre aux deux interrogations suivantes:

- Comment les innovations technologique et organisationnelle évoluent-elles et s'influencent-elles dans le temps?

- Quels sont les mécanismes de diffusion de l'innovation à l'œuvre en matière de technologie et d'organisation?

Dans une première partie, nous montrerons comment l'état actuel des connaissances nous a amenée à formuler ces questions. Nous verrons, dans une deuxième partie, les choix méthodologiques effectués et l'intérêt que présentent les PME pour y répondre. Une troisième partie, enfin, relèvera les principales contributions de notre recherche.

1. Si les travaux s'accordent pour définir l'innovation technologique comme des produits et procédés nouveaux ou améliorés (OCDE, 1997), l'acception de l'innovation organisationnelle apparaît plus discutée. Nous nous référons dans cet article à la définition proposée par Ménard (1994) qui distingue les «innovations de forme», à savoir les changements de structure de plus ou moins grande ampleur, des «innovations internes», qui affectent les routines et les tâches liant les participants.

Revue internationale P.M.E., vol. 19, nº 1, 2006

(C) 2006 - Presses de l'Université du Québec

Édifice Le Delta I, 2875, boul. Laurier, bureau 450, Québec, Québec G1V 2M2 • Tél.: (418) 657-4399 - www.puq.ca

Tiré de: Revue internationale P.M.E., vol. 19, $\mathrm{n}^{\circ}$ 1, sous la direction de Louis Raymond • PME1901N

Tous droits de reproduction, de traduction et d'adaptation réservés 


\section{Technologie et organisation: les deux facettes de l'innovation}

L'objectif de cette première partie est double. Il s'agit tout d'abord de montrer comment la littérature reconnaît progressivement la dépendance mutuelle entre les innovations technologique et organisationnelle, au point d'en faire les deux facettes d'une même réalité que constitue le processus d'innovation dans son ensemble (Van de Ven, 1986). Afin de mieux cerner la réalité de ce processus, nous nous intéresserons, dans un second temps, aux mécanismes de diffusion de ces deux types d'innovation. Les interrogations en suspens dans la littérature nous permettront, à l'issue de cette première partie, de formuler deux questions de recherche qui ont guidé notre réflexion.

\subsection{La reconnaissance progressive de la complémentarité entre innovations}

Si, conformément aux propos de Greenan et al. (1993, p. 28), il est aujourd'hui reconnu que «les innovations organisationnelles vont de pair avec les innovations technologiques», leur complémentarité ne s'est affirmée que tardivement. Une lecture critique des recherches effectuées depuis plus de 40 ans sur les relations potentielles entre innovations technologique et organisationnelle frappe tout d'abord par le nombre considérable d'approches que nous qualifions de «dichotomiques» en cela qu'elles nient la possibilité d'interaction entre ces innovations. Peu à peu remises en cause, elles ouvrent la voie à des approches dites «intégratives».

La contingence (Contingency Approach), qui constitue le premier groupe de travaux significatifs sur les phénomènes d'innovation en entreprise, aborde spécifiquement la question du lien technologie-organisation. Toutefois, ce courant s'intéresse peu à la dynamique organisationnelle qui n'est qu'une conséquence de l'impératif technologique dans le cadre d'une conception causale et unilatérale. Dès les années 1950, l'analyse sociotechnique, à travers le principe «d'optimisation jointe» (joint optimization principle), remet en cause cette analyse et affirme le nécessaire équilibre entre système social et système technique de l'organisation. En introduisant l'idée de réciprocité entre les évolutions des deux systèmes de l'organisation, ce courant offre une nouvelle perspective d'analyse. Les travaux qui en sont issus se limitent pourtant largement à appréhender l'impact des changements techniques sur le système social et ne s'affranchissent finalement que timidement de l'impératif technologique. Il faut donc attendre les années 1970 pour voir se multiplier les recherches qui permettront d'enrichir l'étude des liens entre innovations technologique et organisationnelle. Celles-ci se caractérisent par un point commun fondamental: elles procèdent toutes d'un déplacement d'attention de la technologie vers l'organisation. La technologie cesse d'être appréhendée comme une donnée externe qui, en entrant dans l'organisation la modifie, pour être

Revue internationale P.M.E., vol. 19, n 1, 2006

(C) 2006 - Presses de l'Université du Québec

Édifice Le Delta I, 2875, boul. Laurier, bureau 450, Québec, Québec G1V 2M2 • Tél.: (418) 657-4399 - www.puq.ca

Tiré de: Revue internationale P.M.E., vol. 19, no 1, sous la direction de Louis Raymond • PME1901N

Tous droits de reproduction, de traduction et d'adaptation réservés 
considérée comme le fruit de cette même organisation. Le dilemme énoncé par Daft (1982) contribue grandement à cette évolution théorique. En soulignant que différentes caractéristiques organisationnelles sont requises pour les innovations technologiques ou les innovations organisationnelles, il traite pour la première fois explicitement de la question de la réalisation conjointe d'innovations de différent type. Pour autant, la perspective dichotomique n'est pas dépassée. Des caractéristiques organisationnelles opposées sont en effet jugées favorables à chaque type d'innovation, la mise en œuvre de l'une se faisant au détriment de l'autre'2.

L'ensemble de ces travaux donne toutefois naissance à un vaste domaine de recherche sur l'étude des contextes organisationnels qui favorisent l'adoption ou la diffusion de l'innovation technologique. En montrant que l'évolution des formes organisationnelles facilite le développement de produits ou procédés nouveaux, ils participent à un renouvellement théorique qui nous invite à considérer à la fois les dimensions organisationnelle et technologique de l'innovation. Ce renouveau théorique s'exprime pleinement à partir des années 1990 dans des approches que nous qualifions d' «intégratives». Ces dernières mettent tout d'abord en évidence la coexistence entre innovations technologique et organisationnelle. Ainsi, à l'instar des travaux de Damanpour (1987), Damanpour, Evan et Szabat (1989) ou de Kimberly et Evanisko (1981), plusieurs recherches soulignent l'importance de l'adoption conjointe d'innovations par les organisations pour assurer leur performance ${ }^{3}$. Dans la même veine, les travaux de Greenan et al. (1993) montrent l'importance de l'innovation organisationnelle au sein des entreprises technologiquement innovantes. La dimension temporelle des relations entre innovations fait également l'objet d'une attention particulière. Il s'agit désormais de s'intéresser à l'ordre d'apparition des différentes innovations dans le temps. Plusieurs modèles sont alors proposés. Le premier, de type séquentiel (Damanpour, Evan et Szabat, 1989), souligne l'antériorité de l'innovation technologique sur l'innovation organisationnelle. Le second, dit «modèle d'innovation synchrone (Ettlie, 1988), met en évidence l'adoption simultanée des différentes innovations. Enfin, des analyses de co-évolution (Rosenkopf et Tushman 1994; Van de Ven et Garud 1994) caractérisent des rythmes parallèles d'évolutions technologique et organisationnelle dans une perspective évolutionniste. Quelques travaux complètent cette perspective temporelle pour étudier les influences réciproques entre innovations. À l'instar des travaux d'Henderson et Clark (1990) sur l'innovation architecturale, certaines

2. Ainsi, alors que la centralisation et la formalisation sont jugées favorables à l'innovation organisationnelle, elles nuisent à l'innovation technologique. De même, la complexité, en stimulant la génération d'idées nouvelles, est supposée faciliter l'innovation technologique mais nuire aux innovations en matière d'organisation.

3. Cette adoption conjointe permettrait ainsi d'éviter le «décalage organisationnel» (organizational lag) mis en évidence par Evan (1966). Ce concept désigne précisément l'excès d'innovations technologiques sur les innovations organisationnelles.

Revue internationale P.M.E., vol. 19, nº 1, 2006

(C) 2006 - Presses de l'Université du Québec

Édifice Le Delta I, 2875, boul. Laurier, bureau 450, Québec, Québec G1V 2M2 • Tél.: (418) 657-4399 - www.puq.ca

Tiré de: Revue internationale P.M.E., vol. 19, n 1, sous la direction de Louis Raymond • PME1901N

Tous droits de reproduction, de traduction et d'adaptation réservés 
recherches montrent ainsi que les innovations technologiques conduisent à des modifications organisationnelles. D'autres recherches, bien plus rares, proposent de renverser le point d'entrée théorique et considèrent d'abord les innovations organisationnelles pour souligner leurs effets sur la conduite des développements de produits et procédés. Par sa capacité à modifier la nature et l'agencement des activités et donc à produire un actif humain particulier, l'innovation organisationnelle apparaît alors comme une source essentielle de l'apprentissage collectif qui détermine à son tour une capacité de création et/ou d'absorption technologique (Ménard, 1994). Ce premier volet théorique a mis l'accent sur la complémentarité entre innovations technologique et organisationnelle. L'analyse de leur mécanisme de diffusion respectif enrichit les approches précédentes afin de mieux cerner la complexité du processus d'innovation dans son ensemble.

\subsection{La question de la diffusion des innovations technologique et organisationnelle}

La très grande majorité des travaux dont nous disposons en matière de diffusion se limitent aux évolutions de produits et de procédés. Seuls les travaux de Rogers (1995) font figure d'exception en analysant les mécanismes de diffusion d'innovations de natures variées. Quels éclairages ces recherches jettent-elles sur les innovations organisationnelles?

Les travaux pionniers de Schumpeter ont, dès les années 1930, souligné la nécessité de saisir les processus de diffusion de l'innovation. Ils ont donné lieu à un foisonnement de thématiques aussi bien en gestion, en économie qu'en sociologie. Les synthèses de Alange, Jacobsson et Jarnehammar (1998) et de Teece (1988) mettent l'accent sur la nature cumulative et tacite ainsi que sur l'importance des phénomènes de réseau en matière de technologie. La nature cumulative et tacite des connaissances technologiques est particulièrement bien représentée par l'école évolutionniste (Teece, 1988) qui décrit le développement technologique comme un processus propre à la firme, contraint par des chemins (path dependancy), fruit de variables techniques et économiques indissociables de son passé. Les concepts clés de trajectoires, de routines ou encore de portefeuille de compétences inscrivent les développements technologiques dans les connaissances antérieures. De plus, le caractère fondamentalement tacite des savoirs technologiques conditionne directement la capacité de la firme à exploiter des connaissances externes (Cohen et Levinthal, 1990). L'aptitude à développer de nouveaux produits dépend certes de la R-D interne, mais aussi de «capacités d'absorption» à travers l'entretien d'un «capital humain» capable d'intégrer des développements externes. Cette capacité d'absorption rend dialectique le processus de création et de diffusion des technologies, car l'entreprise doit combiner les savoirs externes avec son propre capital technologique (De Meyer, Dubuisson et Le Bas, 1999). Or, pour y parvenir, elle

Revue internationale P.M.E., vol. 19, nº 1, 2006

(C) 2006 - Presses de l'Université du Québec

Édifice Le Delta I, 2875, boul. Laurier, bureau 450, Québec, Québec G1V 2M2 • Tél.: (418) 657-4399 - www.puq.ca

Tiré de: Revue internationale P.M.E., vol. 19, n 1, sous la direction de Louis Raymond • PME1901N

Tous droits de reproduction, de traduction et d'adaptation réservés 
doit faire face à un double phénomène: d'une part, les capacités d'absorption sont directement contraintes par les connaissances accumulées et, d'autre part, les savoirs intégrés modifient en permanence les savoirs propres à l'organisation.

Le recours aux capacités d'absorption témoigne également de l'importance de l'aptitude de la firme à s'insérer dans un réseau et caractérise donc fortement le processus de diffusion des innovations technologiques. À partir des années 1980, plusieurs travaux ont souligné le rôle clé des réseaux entre firmes comme vecteur de développement de produits (Freeman 1991; Robertson et Langlois, 1995). En France, les travaux du Centre de sociologie de l'innovation ont fortement étudié cette dimension collective de l'innovation à travers le concept de Réseau technico-économique ou RTE (Callon, Laredo et Mustar, 1995). Ils indiquent que l'innovation ne peut être produite que par des ensembles d'acteurs hétérogènes qui participent à son élaboration et à sa diffusion. Von Hippel (1978) a, à ce titre, particulièrement insisté sur le caractère informel des réseaux formés notamment par des ingénieurs de firmes concurrentes, ou encore sur les liens entretenus entre la firme et ses clients potentiels (lead users). Dès lors, comme le relèvent Alange, Jacobsson et Jarnehammar (1998), la littérature reconnaît l'existence d'un «marché» qui, aussi bien en amont (firmes existantes dans le secteur ou dans des secteurs connexes, organismes de valorisation scientifiques, universités, etc.) qu'en aval (clients potentiels), favorise la création et la diffusion des compétences technologiques. Comme le résument ces derniers auteurs, l'innovation technologique peut donc être analysée comme le fruit d'un processus cumulatif et propre à la firme au sein duquel les réseaux jouent un rôle clé pour assurer la diffusion de connaissances tacites.

Si les travaux contemporains font ressortir l'urgence d'appréhender les phénomènes intra-organisationnels dans les processus d'innovation (Ménard, 1994), peu d'auteurs en ont fait un thème d'investigation particulier. La question de la diffusion des innovations organisationnelles, notamment, apparait majeure et paradoxalement presque passée sous silence. C'est donc sous l'angle de la transposition des connaissances acquises en matière d'innovation technologique qu'elle est appréhendée par les travaux les plus récents (Alange, Jacobsson et Jarnehammar, 1998; Rogers, 1995).

Si ces derniers sont d'avis que le caractère tacite des connaissances est déterminant en matière technologique, il l'est encore davantage dès que l'on s'intéresse aux évolutions organisationnelles. Les connaissances liées à l'innovation technologique reposent en effet sur un support matériel et technique (hardware embodied knowledge) alors qu'en matière d'organisation, elles sont souvent rattachées à la personne qui les possède (person embodied knowledge) et, de ce fait, très peu transférables. S'intéressant en particulier à la dynamique d'introduction des

Revue internationale P.M.E., vol. 19, $\mathrm{n}^{\circ} 1,2006$

(C) 2006 - Presses de l'Université du Québec

Édifice Le Delta I, 2875, boul. Laurier, bureau 450, Québec, Québec G1V 2M2 - Tél.: (418) 657-4399 - www.puq.ca

Tiré de: Revue internationale P.M.E., vol. 19, no 1, sous la direction de Louis Raymond • PME1901N

Tous droits de reproduction, de traduction et d'adaptation réservés 
innovations managériales, David (1996) distingue les innovations orientées sur les relations de celles orientées sur les connaissances mettant en évidence l'importance des échanges informels de savoirs liés aux évolutions organisationnelles.

De plus, la nature tacite des connaissances requises pour l'innovation organisationnelle rend problématique leur éventuelle protection posant de fait toute la question de l'incitation au changement organisationnel. Cette question d'incitation vient également de la difficulté à mesurer le «retour sur investissement» lié au changement organisationnel alors qu'il implique souvent pour l'entreprise une perturbation plus importante que le changement technologique. Alter (2000) soutient, à ce propos, que le passage de l'invention à l'innovation repose sur une appropriation de «nouvelles combinaisons» qui ne peut en aucun cas être décrétée. Ce passage est d'autant plus délicat sur le plan organisationnel que les logiques d'innovation et d'organisation sont réputées antagonistes: alors que la première tire parti des incertitudes, la seconde vise à les réduire.

Selon Alange, Jacobsson et Jarnehammar (1998), les caractéristiques de l'innovation organisationnelle introduisent une dernière difficulté quant à sa diffusion qui tient au rôle des réseaux. Les auteurs montrent, en effet, que ces derniers ont une moindre importance dans la diffusion des innovations organisationnelles que dans celle des innovations technologiques. Ils sont en outre très peu formalisés, notamment en raison de la nature tacite des connaissances, comme nous l'avons mentionné précédemment. De plus, il existe peu de collectifs qui, comme avec l'innovation technologique, participent à la création et à la diffusion de pratiques managériales ou formes organisationnelles novatrices. Les travaux de Chanal (2002) soulignent également que le rôle des organismes de conseil en innovation auprès des PME se limite en général aux savoir-faire technologiques. Ce sont surtout les innovations organisationnelles qui souffrent du manque de «fournisseurs» pour assurer leur diffusion, de sorte qu'elles sont encore très largement vouées à être intégrées par des «clients internes» (Alter, 2000).

La lecture critique des travaux sur les liens entre les innovations et leurs mécanismes de diffusion fait émerger un certain nombre d'interrogations qui méritent des approfondissements. Les recherches relatives à la coexistence des innovations possèdent plusieurs limites. Elles soulignent certes l'existence d'un lien entre ces innovations mais ne permettent pas d'en appréhender la nature. Par ailleurs, les travaux centrés sur l'analyse temporelle de leurs relations présentent des modèles différents, voire contradictoires, alternant entre approches séquentielles et simultanées. Au-delà des caractéristiques différentes en matière d'innovations technologique et organisationnelle, les travaux relatifs à la diffusion des innovations nous amènent à réfléchir davantage sur la nature des connaissances qu'exige chaque type d'innovation mais surtout sur les mécanismes d'incitation et les acteurs liés à l'innovation organisationnelle. Dès lors, deux questions clés peuvent être formulées:

Revue internationale P.M.E., vol. 19, n 1, 2006

(C) 2006 - Presses de l'Université du Québec

Édifice Le Delta I, 2875, boul. Laurier, bureau 450, Québec, Québec G1V 2M2 • Tél.: (418) 657-4399 - www.puq.ca

Tiré de: Revue internationale P.M.E., vol. 19, no 1, sous la direction de Louis Raymond • PME1901N

Tous droits de reproduction, de traduction et d'adaptation réservés 
- Comment les innovations technologique et organisationnelle évoluent-elles et s'influencent-elles dans le temps?

- Quels sont les mécanismes de diffusion de l'innovation à l'œuvre en matière de technologie et d'organisation?

\section{2. Éléments de méthodologie}

La démarche retenue est qualitative; elle repose sur trois études de cas de PME technologiquement innovantes.

\subsection{Une approche qualitative centrée sur la réalisation de cas multiples}

Dans le domaine de l'innovation, plusieurs travaux de référence s'appuient sur une démarche qualitative reposant sur des études de cas pour mettre à l'épreuve ou engendrer des théories. Elle apparaît appropriée à la complexité du phénomène étudié et permet d'appréhender à la fois les causalités récursives («le pourquoi») et l'enchaînement des événements dans le temps («le comment»; Yin, 1994). Nous avons privilégié cette stratégie, car elle autorisait à la fois:

- la prise en compte de la dimension temporelle dont l'importance dans la compréhension des phénomènes d'innovation est reconnue par de nombreux auteurs;

- la formulation de premières explications sur les liens entre innovations permettant de dépasser les seules approches statistiques (Einsenhardt, 1989);

- une compréhension fine des mécanismes de diffusion des innovations.

L'étude de cas multiples a été retenue dans le cadre d'une réplication littérale (Yin, 1994), fondée sur la recherche de régularités entre les cas étudiés.

\subsection{Les PME innovantes comme terrain d'investigation}

Si le rôle de la PME dans l'innovation est aujourd'hui reconnu par de nombreux auteurs, l'essentiel des travaux s'est concentré jusqu' au début des années 1980 sur les institutions publiques et surtout les grandes entreprises conformément à la perspective schumpétérienne selon laquelle elles auraient seules les moyens d'innover. C'est notamment autour des travaux de Rothwell (1978) que les avantages de la PME en matière d'innovation ont été relevés. Dans cette même veine, plusieurs enquêtes ont été réalisées sur le rôle de la PME dans la production d'innovations. En France, les travaux du SESSI ont souligné l'importance de l'activité innovatrice

Revue internationale P.M.E., vol. 19, $\mathrm{n}^{\circ} 1,2006$

(C) 2006 - Presses de l'Université du Québec

Édifice Le Delta I, 2875, boul. Laurier, bureau 450, Québec, Québec G1V 2M2 • Tél.: (418) 657-4399 - www.puq.ca

Tiré de: Revue internationale P.M.E., vol. 19, no 1, sous la direction de Louis Raymond - PME1901N

Tous droits de reproduction, de traduction et d'adaptation réservés 
des PME en mettant en évidence un écart important au seuil des 50 puis 200 salariés, ce qui a guidé la sélection de nos cas (Lehoucq, 1994). Les atouts de la PME sont variés. Ils concernent principalement leur capacité de réaction à l'environnement, la souplesse des communications internes, la rapidité des prises de décision, la plus grande proximité au marché et, plus généralement, la dynamique entrepreneuriale résultant d'une bureaucratie moins lourde. En d'autres termes, ce sont des caractéristiques organisationnelles qui favorisent ici l'innovation. Les PME technologiquement innovantes nous sont donc apparues comme un terrain d'investigation privilégié à l'analyse des évolutions organisationnelle et technologique de plus ou moins grande ampleur. Il s'agit, en outre, d'un terrain peu exploré par les travaux centrés sur l'étude des liens entre innovations ainsi que sur leurs mécanismes de diffusion. En tant que terrain novateur, les PME offraient la possibilité d'enrichir considérablement nos connaissances dans ce domaine. Notre recherche repose sur trois études de cas.

Les cas ont dû être sélectionnés avec attention afin de rendre effectivement possible l'étude des interactions entre les dimensions technologique et organisationnelle de l'innovation. Nous avons donc procédé à l'établissement d'un échantillonnage théorique (Yin, 1994). Pour cela, plusieurs critères de sélection ont été établis, après avoir colligé des travaux antérieurs (le Manuel d'Oslo principalement) et consulté divers experts (conseillers technologiques, membres d'agences de développement technologique telles que l'Anvar et l'Arist...). D'un point de vue technologique, les critères de sélection retenus ont été les suivants: secteurs haute ou moyenne technologie selon la classification de l'OCDE reprise dans le Manuel d'Oslo, au moins $10 \%$ du chiffre d'affaires investis en R-D, environ $20 \%$ des effectifs en R-D, dépôt de brevets, au moins un produit développé reconnu comme une innovation radicale dans le secteur. Sur le plan organisationnel, l'établissement de critères de sélection a été plus délicat, car il n'existe pas de critères objectifs représentatifs de l'activité innovante. C'est donc davantage la référence à des «indices» tels que l'existence de groupes de projets, de démarche de certification, d'une politique de communication active, de collaborations externes, etc., qui a été retenue. En outre, la taille de l'entreprise (100 personnes au minimum) et les années d'existence (10 ans) ont été considérées comme garantes d'évolutions en matière d'organisation.

La première société, Pellenc, qui constitue notre cas exploratoire, est spécialisée dans les applications des hautes technologies aux domaines de la viticulture et de l'arboriculture. Détenant plus de 100 brevets dans le monde, elle est un des principaux constructeurs de matériels assistés et automatiques pour la taille des vignes. D'abord spécialisée dans des outils électroportatifs de type sécateur électronique, Pellenc s'est positionnée également sur des équipements lourds à partir de 1993 en proposant des machines à vendanger. D'une organisation fonctionnelle classique, Pellenc est passée récemment à une organisation par ligne de produits, chacune d'entre elles possédant désormais sa propre unité de R-D. La seconde, Istar, produit

Revue internationale P.M.E., vol. 19, nº 1, 2006

(C) 2006 - Presses de l'Université du Québec

Édifice Le Delta I, 2875, boul. Laurier, bureau 450, Québec, Québec G1V 2M2 • Tél.: (418) 657-4399 - www.puq.ca

Tiré de: Revue internationale P.M.E., vol. 19, n 1, sous la direction de Louis Raymond • PME1901N

Tous droits de reproduction, de traduction et d'adaptation réservés 
des données géographiques qui sont essentiellement destinées aux installateurs de téléphonie mobile. Istar a mis au point une méthode de traitement d'images satellite Spot qui permet d'obtenir une représentation du terrain et de l'occupation des sols en trois dimensions. Istar est actuellement le premier fournisseur mondial de données numériques pour les télécommunications. Cette position est le fruit de compétences technologiques mais aussi d'un virage stratégique effectué au milieu des années 1990. Ce dernier a consisté à assurer la production de données «en avance de phase» et à proposer sur le marché des «packages» adaptables de solutions techniques aux opérateurs. La troisième société, Innovtech, conçoit, développe et fabrique des appareils de mesure de température et de pression. Les compétences technologiques acquises en la matière l'ont rapidement amenée à proposer également des systèmes d'alarme et de surveillance. Innovtech offre ainsi actuellement une gamme complète d'appareils de contrôle. Ses produits équipent principalement des navires, mais aussi des usines et centrales électriques en France et à l'étranger.

\subsection{Méthode de recueil et d'analyse des données}

Yin (1994) présente six sources de données mobilisables dans le cadre d'études de cas: la documentation, les archives, l'entretien, l'observation directe, l'observation participante et la simulation. Nous avons eu recours à trois types de sources parmi celles énoncées:

- l'entretien constitue la principale source de nos données. Trente-sept entretiens d'une durée moyenne de deux heures ont été réalisés selon un guide d'entretien. Ce dernier a été établi à l'issue de notre revue de littérature et pendant la démarche d'identification des cas avec l'aide de différents experts rencontrés à cette fin;

- la documentation, notre deuxième source de données, nous a permis de compléter et de corroborer les informations issues des entretiens. Elle a ainsi permis d'améliorer la validité des autres sources;

- l'observation non participante, enfin, est venue compléter le recueil, et ce, principalement avec notre deuxième cas.

Le choix de ces sources a été guidé par les orientations méthodologiques. Nous souhaitions reconstruire des chronologies puis formuler des explications sur les liens entre les innovations. À ce titre, les entretiens nous ont permis d'obtenir une information riche sur des thèmes dont le contenu technique nécessitait souvent des éclaircissements de notre part. Leur nature interactive nous est donc apparue particulièrement favorable au recueil des données. Toutefois, cette source introduit des biais, principalement liés à la mémorisation par les informants. L'utilisation de documents nous est donc apparue comme un moyen privilégié pour limiter ces biais et enrichir les informations obtenues.

Revue internationale P.M.E., vol. 19, nº 1, 2006

(C) 2006 - Presses de l'Université du Québec

Édifice Le Delta I, 2875, boul. Laurier, bureau 450, Québec, Québec G1V 2M2 • Tél.: (418) 657-4399 - www.puq.ca

Tiré de: Revue internationale P.M.E., vol. 19, no 1, sous la direction de Louis Raymond • PME1901N

Tous droits de reproduction, de traduction et d'adaptation réservés 
L'analyse des données repose très largement sur les recommandations de Miles et Huberman (1991). Conformément aux préconisations de ces auteurs, nous avons réalisé une première analyse au cours de notre cas exploratoire (Pellenc). C'est durant cette étape que nous avons établi un codage dit de premier niveau; ce codage a ensuite été enrichi pour aboutir à un codage thématique. La démarche générale d'analyse a reposé sur une analyse intra- puis intersites, afin de dégager des régularités. Différents formats de présentation proposés par Miles et Huberman ont été mobilisés, d'autres, créés pour répondre aux besoins de l'analyse: par exemple, matrice chronologique des différentes innovations, matrice des effets de l'innovation organisationnelle sur l'innovation technologique, matrice des moteurs et des acteurs des différentes innovations.

\section{Principaux résultats et discussion}

Il s'agit à présent de prolonger les travaux existants en présentant les éléments de contribution à nos deux questions de recherche. Nous montrerons comment l'analyse de nos cas nous permet de faire ressortir la complémentarité entre innovations technologique et organisationnelle, d'une part, et de caractériser des mécanismes de diffusion pour chacune d'entre elles, d'autre part.

\subsection{Une interaction croissante entre innovations technologique et organisationnelle}

L'interdépendance entre les innovations est le fruit d'une double analyse. Cette dernière a consisté tout d'abord à repérer dans une perspective chronologique les différentes innovations réalisées dans le temps afin d'étudier ensuite leurs influences mutuelles.

Au sein de chaque cas, une étude détaillée des différents types d'innovations réalisées sur longue période a été effectuée. Cette analyse nous a permis de relever trois phases clés, caractéristiques de l'évolution chronologique des dimensions technologique et organisationnelle de l'innovation (on trouvera en annexe une métamatrice des différentes innovations dans l'ensemble des cas).

La première phase est marquée par la seule présence d'innovations technologiques; celles-ci sont de type radical et correspondent à des premières sur le marché.

Les premiers produits qui sont sortis étaient des produits sans concurrence, personne ne proposait de produits similaires (directeur général, Pellenc).

Sur le plan organisationnel, les évolutions au cours de cette première période concernent essentiellement la mise en place d'unités de production. Nous sommes en présence d'un fonctionnement extrêmement simple, l'entrepreneur-innovateur assumant à la fois la direction de la société et le développement des produits.

Revue internationale P.M.E., vol. 19, nº 1, 2006

(C) 2006 - Presses de l'Université du Québec

Édifice Le Delta I, 2875, boul. Laurier, bureau 450, Québec, Québec G1V 2M2 • Tél.: (418) 657-4399 - www.puq.ca

Tiré de: Revue internationale P.M.E., vol. 19, n 1, sous la direction de Louis Raymond • PME1901N

Tous droits de reproduction, de traduction et d'adaptation réservés 
Avec la croissance de la société, on s'est organisés. Il s'agissait davantage d'adaptations. Je ne comparerais pas cela à la mise en place des Domaines d'Activités Stratégiques qui a été une vraie initiative aux conséquences importantes sur notre fonctionnement (responsable Développement, Innovtech).

La deuxième phase est caractérisée par la coexistence de diverses innovations technologiques et organisationnelles. Les innovations technologiques réalisées au cours de cette période concernent principalement des améliorations de l'existant. Il s'agit très largement d'applications à de nouveaux contextes plutôt que de produits véritablement novateurs. Elles relèvent par ailleurs essentiellement de l'innovation de procédé, destinée à assurer la fabrication industrielle des produits mis au point au cours de la première phase.

Des outils ont été améliorés, mais il n'y a pas eu de véritable innovation technologique. Notre point fort à ce moment-là a concerné plutôt la production et le technicocommercial... La standardisation a surtout conduit à des adaptations de l'outil de production (responsable Développement, Istar).

D'un point de vue organisationnel, c'est au cours de cette deuxième phase que sont introduites les premières évolutions significatives; celles-ci concernent principalement la création de départements marketing et l'instauration de démarches qualité.

Mettre en place une compétence marketing dans une PME de 14 millions de francs avec des produits qui n'étaient pas encore standardisés, c'était quelque chose de vraiment nouveau (directeur général, Istar).

De plus, l'arrivée de cadres aux compétences variées dans l'entreprise s'accompagne d'une séparation entre les activités de direction et les activités de recherche, auparavant assumées par le seul entrepreneur.

La troisième phase, enfin, est toujours caractérisée par la coexistence d'innovations technologiques et organisationnelles. Une évolution notable apparaît cependant en matière d'innovation technologique par rapport à la période antérieure puisque les trois sociétés renouent avec des innovations de type radical.

Les robots que l'on a commercialisés au cours des deux dernières années sont de véritables innovations. Ils reposent sur des technologies uniques, brevetées sur le marché (responsable Automatisme, Pellenc).

Sur le plan de l'organisation, les innovations sont plus nombreuses. De plus ou moins grande ampleur, elles concernent aussi bien la structure globale de la société (par exemple, la mise en place d'une organisation par Domaines d'Activités Stratégiques au sein d'Innovtech) que certaines unités (par exemple, l'intégration des clients dans la mise au point des produits chez Istar).

Revue internationale P.M.E., vol. 19, nº 1, 2006

(C) 2006 - Presses de l'Université du Québec

Édifice Le Delta I, 2875, boul. Laurier, bureau 450, Québec, Québec G1V 2M2 • Tél.: (418) 657-4399 - www.puq.ca

Tiré de: Revue internationale P.M.E., vol. 19, no 1, sous la direction de Louis Raymond • PME1901N

Tous droits de reproduction, de traduction et d'adaptation réservés 
Les enseignements relatifs à la chronologie des différents types d'innovations viennent enrichir la littérature existante en la matière. Peu de travaux se sont, en effet, centrés sur l'évolution temporelle des innovations réalisées par la firme. La tendance successorale mise en évidence par Abernathy et Utterback (1978) ne prend en compte que les innovations technologiques pour appréhender l'ordre d'apparition des innovations de produits et procédés. Les travaux ayant introduit la dynamique d'innovation organisationnelle sont majoritairement inscrits dans une perspective d'analyse sectorielle (Rosenkopf et Tushman, 1994; Van de Ven et Garud, 1994). Ceux ayant fait de l'organisation leur unité d'analyse ne se sont pas penchés sur la PME et aboutissent à des résultats contradictoires alternant entre approche séquentielle ou simultanée. Notre recherche offre donc une contribution à la mise en perspective temporelle des différentes innovations réalisées par les PME sur longue période. Ainsi, sur le long terme, nos résultats montrent l'antériorité de l'innovation technologique sur l'innovation organisationnelle. Toutefois, la pertinence de cette approche séquentielle est rapidement remise en cause dans les deuxième et troisième phases mentionnées, toutes deux caractérisées par la réalisation conjointe d'innovations. Cette première contribution a été approfondie par l'analyse de leurs influences mutuelles.

Pour souligner les liens de réciprocité entre les innovations, nous nous sommes livrée à un examen des «moteurs». Cette analyse a mis en relief le rôle inducteur de l'innovation technologique et la fonction de support de l'innovation organisationnelle.

L'étude des différents moteurs de l'innovation organisationnelle a souligné l'importance de l'innovation technologique comme un de ses facteurs déclenchants. Cette dernière a donc été qualifiée «d'inducteur» en ce sens qu' elle «déclenche», «conduit à» ou encore «engendre» des innovations sur le plan organisationnel. Il convient cependant de noter que, durant les deux premières phases décrites précédemment, les innovations organisationnelles sont le fait de réorientations stratégiques, du développement de l'activité ou de dysfonctionnements internes. Ce rôle inducteur de l'innovation technologique n'apparaît sur l'ensemble des cas qu'au cours de la troisième phase. On assiste, par exemple, à la création de fonctions ou de comités voués à l'innovation technologique:

La fonction chef de produit a été développée essentiellement pour susciter l'innovation technologique (responsable Marketing, Istar).

Le comité Nouveaux Produits a pour vocation à pousser à l'innovation, c'est typiquement une organisation dont l'objectif est de favoriser les développements technologiques (responsable Développement, Innovtech).

L'analyse de l'influence de l'innovation organisationnelle sur l'innovation technologique, quant à elle, a permis de mettre en évidence son rôle de «support» ou «facilitateur» des développements de produits ou procédés nouveaux. Cette

Revue internationale P.M.E., vol. 19, nº 1, 2006

(C) 2006 - Presses de l'Université du Québec

Édifice Le Delta I, 2875, boul. Laurier, bureau 450, Québec, Québec G1V 2M2 • Tél.: (418) 657-4399 - www.puq.ca

Tiré de: Revue internationale P.M.E., vol. 19, no 1, sous la direction de Louis Raymond • PME1901N

Tous droits de reproduction, de traduction et d'adaptation réservés 
fonction de support a alors été déclinée en différents phénomènes qu'autorise l'innovation organisationnelle: la spécialisation des activités de recherche, une meilleure connaissance des attentes du marché, la formalisation de processus et développement et, enfin, la création de conditions d'apprentissage entre les membres de l'organisation. On trouvera dans le tableau suivant quelques illustrations de cette déclinaison.

TABleau 1

Le rôle du support de l'innovation organisationnelle dans la conduite des développements de produits: quelques exemples

\begin{tabular}{|c|c|c|}
\hline $\begin{array}{l}\text { Déclinaisons } \\
\text { possibles de la } \\
\text { fonction de support }\end{array}$ & $\begin{array}{l}\text { Exemples } \\
\text { d'innovations } \\
\text { organisationnelles } \\
\text { concernées }\end{array}$ & Extraits d'entretien \\
\hline $\begin{array}{l}\text { Spécialisation des } \\
\text { activités } \\
\text { de recherche }\end{array}$ & $\begin{array}{l}\text { Instauration d'une } \\
\text { organisation par } \\
\text { ligne de produit. }\end{array}$ & $\begin{array}{l}\text { «Désormais, chaque département } \\
\text { possède son unité de recherche et gère } \\
\text { ses développements de manière auto- } \\
\text { nome. Seul un département, en raison de } \\
\text { ses compétences transversales, conserve } \\
\text { un rôle de service vis-à-vis des autres } \\
\text { unités de recherche» (PDG, Pellenc). }\end{array}$ \\
\hline $\begin{array}{l}\text { Meilleure } \\
\text { connaissance } \\
\text { du marché }\end{array}$ & $\begin{array}{l}\text { Instauration de } \\
\text { Domaines d'Acti- } \\
\text { vités Stratégiques. }\end{array}$ & $\begin{array}{l}\text { «Travailler par marché permet aux gens } \\
\text { d'être un peu plus curieux de ce qu'ils } \\
\text { veulent vendre à leurs clients. Il devrait } \\
\text { en découler de nouveaux produits» } \\
\text { (responsable Développement, Innovtech). }\end{array}$ \\
\hline $\begin{array}{l}\text { Amélioration } \\
\text { des processus de } \\
\text { développement }\end{array}$ & $\begin{array}{l}\text { Instauration d'un } \\
\text { comité Nouveaux } \\
\text { Produits. }\end{array}$ & $\begin{array}{l}\text { «On ne développe pas forcément plus } \\
\text { mais mieux, car on formalise les choses. } \\
\text { On s'oblige à faire des études de marché, } \\
\text { à rédiger des cahiers des charges. Surtout } \\
\text { a priori, on ne peut plus perdre de projet» } \\
\text { (responsable Développement, Innovtech). }\end{array}$ \\
\hline $\begin{array}{l}\text { Création de } \\
\text { conditions } \\
\text { d'apprentissage } \\
\text { collectif }\end{array}$ & $\begin{array}{l}\text { Création d'équipes } \\
\text { d'intégration. }\end{array}$ & $\begin{array}{l}\text { «Jusqu'à présent, le marketing était } \\
\text { essentiellement de type opérationnel. } \\
\text { Nous n'avions pas pris part de façon } \\
\text { décisive à la gestation de nouveaux } \\
\text { produits. Désormais, c'est différent, il } \\
\text { y a des discussions, des échanges entre } \\
\text { personnes de différents départements, } \\
\text { à un partage de savoirs» (responsable } \\
\text { Marketing, Istar). }\end{array}$ \\
\hline
\end{tabular}

L'analyse des liens de réciprocité entre les innovations technologique et organisationnelle a permis de préciser la nature des influences mutuelles entre innovations, ce que n'autorisait pas la littérature existante, limitée à la mise en évidence

Revue internationale P.M.E., vol. 19, $\mathrm{n}^{\circ}$ 1, 2006

(C) 2006 - Presses de l'Université du Québec

Édifice Le Delta I, 2875, boul. Laurier, bureau 450, Québec, Québec G1V 2M2 • Tél.: (418) 657-4399 - www.puq.ca

Tiré de: Revue internationale P.M.E., vol. 19, n 1, sous la direction de Louis Raymond • PME1901N

Tous droits de reproduction, de traduction et d'adaptation réservés 
de leur coexistence. En mettant en évidence leur rôle respectif d'inducteur et de support, nos résultats nous amènent à privilégier une approche en termes d'interaction, ou encore de «co-activation», en ce sens qu'innovations technologique et organisationnelle s'entretiennent mutuellement. Après avoir retracé l'évolution temporelle des différentes innovations ainsi que la nature de leurs liens, nous avons souhaité mettre davantage l'accent sur leurs mécanismes de diffusion.

\subsection{Des mécanismes de diffusion contrastés entre technologie et organisation}

Conformément à la grille de lecture fournie par la littérature, nous nous sommes intéressée à la nature des connaissances mobilisées ainsi qu'aux effets de réseau pour appréhender les mécanismes de diffusion relatifs aux innovations technologique et organisationnelle.

En matière de technologie, tout d'abord, il convient de noter qu'au-delà du simple caractère tacite de la connaissance, c'est davantage, pour reprendre les termes de Reix (1995), son caractère «équivoque» qui est apparu. La dimension tacite est manifeste à travers des savoirs non formalisés qui font partie de l'identité de l'entreprise.

Au sein de la R-D, les échanges sont surtout informels; en principe tous les membres de l'équipe ont une très bonne connaissance de notre savoir-faire; il y a certes des spécialistes, mais ils ont des connaissances communes, car ils interviennent sur des projets variés (responsable scientifique, Istar).

Toutefois, dans l'ensemble des cas, nous constatons une évolution vers une volonté clairement affichée de formalisation des connaissances technologiques. Celle-ci apparaît principalement par l'instauration de système de veille technologique et l'instauration de démarches de développement de produits de plus en plus standardisée.

Nous avons un souci constant d'amélioration de nos démarches de développement. Aujourd'hui, grâce à l'instauration du comité Nouveaux Produits, nous ne développons pas forcément plus, mais mieux (responsable Développement, Innovtech).

Nos études de cas mettent par ailleurs en évidence le rôle clé de réseaux d'acteurs, tant externes qu'internes qui, réunissant régulièrement des experts de diverses spécialités, constitue un élément déterminant de la capacité de la firme à mettre au point des produits ou des procédés nouveaux. La constitution de ces collectifs d'innovation apparaît dans nos cas à différents niveaux. Elle se concrétise tout d'abord par l'existence de liens avec des organismes de recherche ou de valorisation scientifique dont sont très largement issus les membres de l'organisation. Elle apparaît également à travers l'importance de la mobilité du personnel de recherche, rejoignant ainsi les travaux de Cohen et Levinthal (1990) qui soutiennent que la

Revue internationale P.M.E., vol. 19, nº 1, 2006

(C) 2006 - Presses de l'Université du Québec

Édifice Le Delta I, 2875, boul. Laurier, bureau 450, Québec, Québec G1V 2M2 • Tél.: (418) 657-4399 - www.puq.ca

Tiré de: Revue internationale P.M.E., vol. 19, n 1, sous la direction de Louis Raymond • PME1901N

Tous droits de reproduction, de traduction et d'adaptation réservés 
capacité d'innovation technologique dépend de la R-D, mais aussi de l'entretien d'un capital humain capable d'intégrer des connaissances externes. Pour y parvenir, l'organisation doit certes se doter d'un personnel qualifié (filtrage à l'embauche, formation, etc.), mais elle doit aussi reconnaître l'importance du renouvellement du personnel comme soutien de l'innovation technologique. Dans nos cas, nous avons été témoin d'une forte mobilité des ingénieurs et des scientifiques. La situation du chef de produit d'Istar nous paraît tout à fait révélatrice. Interrogé sur ses fonctions précédentes au sein de la société, celui-ci nous indique:

Il y a deux ans, le département Marketing a été créé; aujourd'hui, j'appartiens à ce département; cela a tellement bougé... j'ai moi-même du mal à suivre... je ne sais plus très bien dans quelle fonction je suis rentré mais $\mathrm{j}$ 'ai maintenant une très bonne connaissance de l'entreprise et de ses marchés.

Ce renouvellement des fonctions permet aux acteurs d'acquérir des savoirfaire de plus en plus transversaux et favorise les remises en cause, condition de l'innovation technologique.

Que ce soit en matière d'organisation ou de technologie, innover, pour moi, c'est être obligé de se remettre en question; dans tous les cas, vous avez un problème à résoudre, vous devez enlever les a priori et reconstruire. Les choses apprises sont à la fois une richesse et un guide. Il faut savoir les utiliser, mais aussi s'en détacher. C'est cet esprit qu'il faut maintenir (responsable Développement Innovtech).

Au-delà des reconfigurations qui créent un environnement interne favorable à l'innovation, nous relevons l'existence et la création régulière d'entités ou supports organisationnels dédiés aux développements technologiques. Plus ou moins formalisés, plus ou moins temporaires, intégrant parfois des acteurs externes, ces entités peuvent être:

- un département de R-D autonome ou des unités de R-D intégrées à une organisation par produit. Chaque entreprise étudiée possède des structures consacrées aux activités de R-D. Il s'agit d'unités de R-D intégrées dans chaque département pour Pellenc. Innovtech possède des unités de R-D pour chaque domaine d'activités stratégiques auxquelles s'ajoute un département de R-D commun qui gère des développements particuliers. Dans le cas d'Istar, le département de R-D est commun à l'ensemble des activités;

- des équipes pluridisciplinaires temporaires liées au développement d'un produit. Dans l'ensemble des cas étudiés, des fonctionnements de type management par projet ont été instaurés. Reposant sur l'intégration des différentes fonctions de l'entreprise, mais aussi de clients potentiels, cette démarche est utilisée dès la première phase de spécification du produit;

Revue internationale P.M.E., vol. 19, $\mathrm{n}^{\circ} 1,2006$

(C) 2006 - Presses de l'Université du Québec

Édifice Le Delta I, 2875, boul. Laurier, bureau 450, Québec, Québec G1V 2M2 • Tél.: (418) 657-4399 - www.puq.ca

Tiré de: Revue internationale P.M.E., vol. 19, no 1, sous la direction de Louis Raymond - PME1901N

Tous droits de reproduction, de traduction et d'adaptation réservés 
- des comités pluridisciplinaires non liés au développement d'un produit spécifique. Ces instances ont été mises en évidence dans notre troisième cas, Innovtech. Il s'agit d'un comité Nouveaux Produits (CNP), d'une part, et d'un comité Marketing (CM), d'autre part. Le CNP a introduit une nouvelle démarche de développement. Constitué de neuf membres occupant des fonctions très diverses, ce comité assure un rôle de sélection et de suivi des projets. À travers l'instauration d'un système dit de «fiches d'enjeu», il examine les projets soumis. Ces fiches constituent en quelque sorte un «réservoir d'innovations» régulièrement passé en revue. Chaque projet retenu reçoit ainsi l'approbation de l'ensemble des membres du CNP qui en suit par ailleurs l'état d'avancement. Le CM complète le rôle du CNP; il est formé par cinq membres (responsable des domaines d'activité stratégiques et responsable marketing). Il est à noter que le responsable R-D n'en fait pas partie. La création du $\mathrm{CM}$ correspond à une volonté de mieux intégrer les attentes du marché dans la démarche de développement de produits. Il effectue une première sélection des fiches d'enjeu soumises par la suite au CNP.

Il convient de signaler par ailleurs que la direction générale joue un rôle clé au sein de ces différentes entités. Conformément aux travaux de Lefebvre (1991) ou encore de Brimm (1984), la formation ainsi que l'attitude proactive des dirigeants à l'égard des évolutions technologiques semblent expliquer cette implication intense de la direction dans la conduite des innovations technologiques. Nous constatons donc que l'innovation technologique repose sur des acteurs et des entités dont le rôle est légitimé par l'organisation.

En matière d'innovation organisationnelle, la nature tacite des connaissances est apparue encore plus significative. Nous avons découvert que les principales évolutions en la matière sont portées par quelques acteurs clés qui, pour ce faire, utilisent leur expérience, voire leur intuition.

Lorsque nous avons mis en place l'organisation par domaines d'activités stratégiques, nous avons réfléchi à la structure, mais notre démarche a été très intuitive, j'ai calqué ce que j'avais observé ailleurs (directeur général, Innovtech).

L'intégration des clients au départ était très peu structurée, c'était très empirique comme méthode, nous nous sommes débrouillés en tirant le meilleur de chacun, nous avons appris et compris en travaillant (responsable Marketing, Istar).

Au-delà de la nature tacite des connaissances, c'est surtout la faiblesse, voire l'absence d'entités ou de supports similaires à ceux dédiés à l'innovation technologique qui est apparue révélatrice. Cette faiblesse entraîne, selon nous, trois conséquences: le recours à des consultants pour enclencher des évolutions organisationnelles, l'engagement encore plus fort de la direction dans la conduite des innovations, la «naissance» des innovations organisationnelles au sein d'entités initialement dédiées aux évolutions technologiques.

Revue internationale P.M.E., vol. 19, nº 1, 2006

(C) 2006 - Presses de l'Université du Québec

Édifice Le Delta I, 2875, boul. Laurier, bureau 450, Québec, Québec G1V 2M2 • Tél.: (418) 657-4399 - www.puq.ca

Tiré de: Revue internationale P.M.E., vol. 19, no 1, sous la direction de Louis Raymond • PME1901N

Tous droits de reproduction, de traduction et d'adaptation réservés 
L'absence d'entités ou d'acteurs spécialisés en matière d'innovation organisationnelle explique largement le recours à des consultants externes. L'ensemble des entreprises étudiées ont, en effet, eu recours à un cabinet pour mener à bien des innovations en termes d'organisation (démarche qualité au sein de Pellenc, instauration d'un nouveau système de communication au sein d'Istar, amélioration des procédures de développement de produits et mise en place des domaines d'activités stratégiques au sein d'Innovtech). Les travaux de Paturel et Barriol (1999) ont, à ce titre, souligné l'importance de conseils externes dans l'instauration d'innovation de gestion dans le domaine de la qualité. Conformément aux travaux de Alange, Jacobsson et Jarnehammar (1998), ces cabinets apparaissent comme des «fournisseurs» d'innovations et participent à leur diffusion. Ils proposent effectivement un «produit» qui, avec plus ou moins de succès, sera mis en œuvre par l'organisation. Toutefois, comme le mentionnent ces auteurs, le rôle des consultants demeure limité en raison même du caractère tacite des connaissances requises.

Nous avons précédemment relevé la forte implication de la direction dans la conduite des innovations technologiques. Celle-ci apparait encore plus importante en matière d'organisation, la direction étant impliquée ici dans l'ensemble du processus d'innovation (de l'idée à la mise en œuvre).

On peut dire qu'en matière de choix d'organisation, tout comme pour les produits et même certainement davantage, la direction a un rôle prépondérant (responsable Département outils électroportatifs, Pellenc).

Les évolutions dans l'organisation ont été essentiellement des décisions de direction générale. Certaines sont d'ailleurs perçues comme des «opérations coup de poing», mais il faut reconnaître que l'on évolue là-dessus depuis quelque temps (responsable Achats, Innovtech).

La position hiérarchique des acteurs apparaît donc déterminante sur le plan organisationnel. Nous retrouvons ici l'importance du phénomène d'institutionnalisation soulignée par Alter (2000). L'auteur montre, en effet, que la diffusion des innovations organisationnelles diffère de celle d'un bien sur un marché et suppose un rôle clé de la direction décrite comme un «superacteur» qui assure son institutionnalisation. De ce fait, le développement d'une innovation organisationnelle ne repose pas nécessairement sur sa qualité intrinsèque mais bien sur sa capacité d'intégration, elle-même liée au pouvoir hiérarchique.

Le dernier élément qui mérite notre attention concerne les entités et les acteurs qui, autres que la direction générale, participent également à la forte imbrication entre innovations au sein de nos cas. L'analyse des différents acteurs de l'innovation indique qu'ils sont nombreux à être impliqués à la fois dans les innovations technologique et organisationnelle. Par exemple, au sein d'Istar, nous pouvons remarquer que les mêmes acteurs participent à l'instauration d'une démarche d'intégration des clients et au développement du produit haute résolution. De même, chez Pellenc

Revue internationale P.M.E., vol. 19, $\mathrm{n}^{\circ} 1,2006$

(C) 2006 - Presses de l'Université du Québec

Édifice Le Delta I, 2875, boul. Laurier, bureau 450, Québec, Québec G1V 2M2 • Tél.: (418) 657-4399 - www.puq.ca

Tiré de: Revue internationale P.M.E., vol. 19, no 1, sous la direction de Louis Raymond - PME1901N

Tous droits de reproduction, de traduction et d'adaptation réservés 
ou Innovtech, on constate que les actuels responsables de départements ont joué un rôle prépondérant dans l'instauration d'une organisation par lignes de produits et par Domaines d'Activités Stratégiques. Or, il s'agit aussi de personnes qui travaillent directement au développement de produits. L'innovation organisationnelle apparaît donc intimement liée aux acteurs mêmes de l'innovation technologique. Dès lors, on assiste parfois à la «production» et à la diffusion d'innovations organisationnelles par les entités vouées d'abord à l'innovation technologique. Cela est particulièrement manifeste dans le cadre de l'instauration du comité Marketing au sein d'Innovtech. Créé pour améliorer le fonctionnement du comité Nouveaux Produits, il est le fruit des réflexions menées en son sein. Des réflexions relatives à l'instauration de «fiches organisationnelles» en sont en effet issues. Calquées sur le mode de fonctionnement des «fiches d'enjeu» dédiées à l'innovation technologique, celles-ci devraient concerner l'ensemble des dysfonctionnements internes. Cette «diffusion» d'innovations organisationnelles par des acteurs ou des entités dédiés à l'innovation technologique nous est apparue particulièrement importante pour rendre compte de leur imbrication effective au sein des entreprises étudiées.

\section{Conclusion}

Cette recherche visait à améliorer la compréhension des processus d'innovation au sein de PME en mettant l'accent sur deux axes insuffisamment abordés par la littérature existante: les influences mutuelles entre les innovations technologique et organisationnelle, d'une part, et les mécanismes de diffusion relatifs à chaque type d'innovation, d'autre part.

Nos résultats enrichissent tout d'abord les travaux relatifs aux différentes innovations réalisées sur longue période qui n'étaient pas jusqu'à présent centrés sur la PME. La perspective longitudinale a permis de relever trois phases caractéristiques de l'évolution des différentes innovations: des innovations technologiques seules, la coexistence, puis l'interaction entre les innovations technologique et organisationnelle. Par ailleurs, rares sont les recherches qui, inscrites dans une approche que nous avons qualifiée «d'intégrative», ont pour objet d'analyse les liens entre les innovations. Si elles admettent effectivement la possibilité d'influences mutuelles entre ces innovations, elles ne permettent pas, en revanche, d'en préciser la nature. En soulignant le rôle d'inducteur de l'innovation technologique et de support de l'innovation organisationnelle, nos résultats caractérisent deux modes particuliers de «co-activation». Ces phénomènes de «co-activation» ont été enrichis par l'analyse comparative de leurs mécanismes de diffusion respectifs. En matière de technologie, nous avons mis en évidence 1) l'importance de la coexistence de connaissances tacites et de démarches de formalisations des savoirs, 2) l'existence de réseaux d'acteurs structurés, tant internes qu'externes, 3) l'entretien de capacité d'absorption, notamment à travers la mobilité du personnel de recherche et 4) la

Revue internationale P.M.E., vol. 19, $\mathrm{n}^{\circ}$ 1, 2006

(C) 2006 - Presses de l'Université du Québec

Édifice Le Delta I, 2875, boul. Laurier, bureau 450, Québec, Québec G1V 2M2 • Tél.: (418) 657-4399 - www.puq.ca

Tiré de: Revue internationale P.M.E., vol. 19, n 1, sous la direction de Louis Raymond • PME1901N

Tous droits de reproduction, de traduction et d'adaptation réservés 
mise en place de supports organisationnels dédiés aux développements technologiques. Concernant la diffusion des innovations organisationnelles, ce sont la nature fondamentalement tacite des connaissances ainsi que l'absence de supports qui sont apparues significatives. Toutefois, au-delà de ces différences, le détour par les connaissances et/ou les acteurs concernés par chaque type d'innovation nous a permis de souligner l'importance des choix opérés en matière de technologie dans les évolutions organisationnelles. Ainsi, pour reprendre le thème de la dépendance de sentier cher aux analyses évolutionnistes, il semble que ce dernier s'applique non seulement au champ respectif de la technologie et de l'organisation, mais qu'il permette plus largement de rendre compte de leur imbrication.

Ces premiers résultats appellent à des approfondissements. Une première voie de recherche consisterait à étendre la démarche suivie à de nouveaux terrains d'investigation afin d'en améliorer la validité externe. Une seconde voie résiderait dans la prise en compte de la réalisation conjointe d'innovations et de ses liens avec la performance. Explicitement relevée dans certains travaux, cette question n'a pas été abordée ici alors que celle de la relation entre performance et innovation apparaît cruciale (Foray et Mairesse, 1999). Enfin, la nature des liens entre technologie et organisation pourrait être envisagée, non seulement dans le cas de la création de technologies nouvelles, comme c'est le cas dans cette recherche, mais aussi dans le cadre de l'implantation de technologies (TIC par exemple). D'un point de vue managérial, ce travail nous invite à attirer l'attention des dirigeants soucieux d'améliorer le développement de leur produit sur l'importance de la réalisation conjointe d'innovations organisationnelles. Les structures d'aide à l'innovation pour les PME sont également fortement concernées par ces résultats qui les incitent à élargir leur politique de soutien trop souvent limitée à des aspects technologiques (Chanal, 2002). Ces prolongements permettraient, en partie au moins, d'améliorer la validité externe des résultats énoncés. En effet, cette recherche s'inscrit dans un contexte de généralisation analytique reposant sur une logique de réplication littérale. Inhérente à l'étude de cas, cette limite incite à adopter des méthodologies complémentaires telles que l'observation qui permettrait en outre de dépasser le caractère longitudinal et rétrospectif de ce travail. 


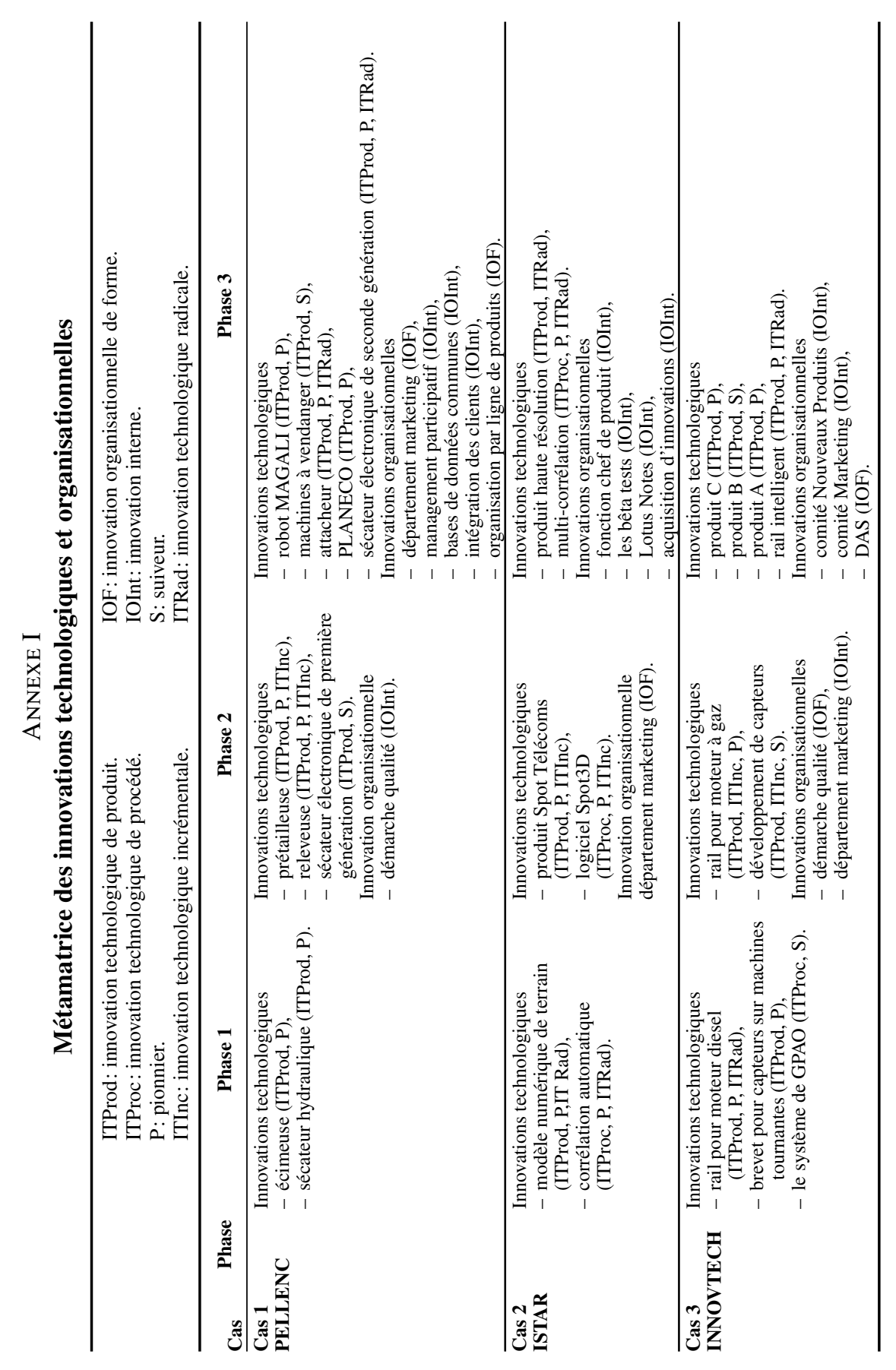

Revue internationale P.M.E., vol. 19, nº 1, 2006

(C) 2006 - Presses de l'Université du Québec

Édifice Le Delta I, 2875, boul. Laurier, bureau 450, Québec, Québec G1V 2M2 • Tél.: (418) 657-4399 - www.puq.ca

Tiré de: Revue internationale P.M.E., vol. 19, n 1, sous la direction de Louis Raymond • PME1901N Tous droits de reproduction, de traduction et d'adaptation réservés 


\section{Bibliographie}

Abernathy, W.J. et J.M. UtTERBACK (1978), «Patterns of industrial innovation», Technology Review, juin-juillet, p. 41-48.

Alange, S., S. Jacobsson et A. Jarnehammar (1998), «Some aspects of an analytical framework for studying the diffusion of organizational innovations», Technology Analysis and Strategic Management, vol. 10, p. 3-21.

Alter, N. (2000), L'innovation ordinaire, Paris, Presses universitaires de France.

BRIMM, M. (1984), «La diffusion des innovations en matière d'organisation», Revue française de gestion, vol. 44, p. 68-79.

Callon, M., P. LARedo et P. Mustar (dir.) (1995), La gestion stratégique de la recherche et de la technologie, Paris, Economica.

Chanal, V. (2002), «Comment accompagner les PME-PMI dans leur processus d'innovation?», Actes de la XI ${ }^{e}$ Conférence internationale de Management stratégique, Paris, juin.

Cohen, W.M. et D.A. Levinthal (1990), «Absorptive capacity: a new perspective on learning and innovation», Administrative Science Quarterly, vol. 35, p. 128-152.

DAFT, R.L. (1982), «Bureaucratic versus nonbureaucratic structure and the process of innovation and change», dans S.B. Bacharach (dir.), Research in the Sociology of Organizations, Greenwich, JAI Press, vol. 1, p. 129-166.

Damanpour, F. (1987), «The adoption of technological, administrative and ancillary innovations: impact of organizational factors», Journal of Management, vol. 13, p. 675-688.

Damanpour, F., W.M. Evan et K.A. Szabat (1989), «The relationship between types of innovation and organizational performance», Journal of Management Studies, vol. 26, p. 587-601.

David, A. (1996), «Structure et dynamique des innovations managériales», Actes de la $V^{k}$ Conférence internationale de Management stratégique, Lille, mai.

De Meyer, A., S. Dubuisson et C. Le Bas (1999), «La thématique des compétences. Une confrontation de points de vue disciplinaires», dans D. Foray et J. Mairesse (dir.), Innovations et Performances, Paris, Éditions de l'École des hautes études en sciences sociales.

EISENHARDT, K. (1989), «Building theories from case study research», Academy of Management Review, vol. 14, p. 532-550.

EtTlie, J.E. (1988), Taking Charge of Manufacturing, How Companies Are Combining Technological and Organizational Innovations to Compete Successfully, San Francisco, Jossey-Bass.

Evan, W.M. (1966), «Organization lag», Human Organizations, vol. 25, p. 51-53.

ForAY, D. et J. MAIRESSE (dir.) (1999), Innovation et performances, Paris, École des hautes études en sciences sociales.

Revue internationale P.M.E., vol. 19, $\mathrm{n}^{\circ} 1,2006$

(C) 2006 - Presses de l'Université du Québec

Édifice Le Delta I, 2875, boul. Laurier, bureau 450, Québec, Québec G1V 2M2 • Tél.: (418) 657-4399 - www.puq.ca

Tiré de: Revue internationale P.M.E., vol. 19, n 1, sous la direction de Louis Raymond • PME1901N

Tous droits de reproduction, de traduction et d'adaptation réservés 
Freeman, C. (1991), «Networks of innovators: a synthesis of research issues», Research Policy, vol. 20, n 5, oct., p. 499-514.

Greenan, N., D. Guellec, G. Broussaudier et L. Miotti (1993), «Innovation organisationnelle, dynamisme technologique et performance des entreprises», INSEE, document de travail G9304, avril.

Henderson, R.M. et K.B. Clark (1990), «Architectural innovation: the reconfiguration of existing product technologies and the failure of established firms », Administrative Science Quarterly, vol. 35, p. 9-30.

Kimberly, J.R. et M.J. Evanisko (1981), «Organizational innovation: the influence of individual, organizational and contextual factors on hospital adoption of technological and administrative innovations», Academy of Management Journal, vol. 24, $\mathrm{n}^{\circ} 4$, p. 689-713.

Lefebvre, E. (1991), «Profit distinctif des dirigeants de PME innovatrices», Revue internationale PME, vol. 4, $\mathrm{n}^{\circ}$ 3, p. 7-26.

LeHoucQ, T. (1994), «Difficultés à l'innovation pour les PMI», dans L'innovation technologique dans l'industrie, Ministère de l'Industrie, Dunod, p. 71-76.

MÉNARD, C. (1994), «La nature de l'innovation organisationnelle», Revue d'économie industrielle, numéro exceptionnel «Économie industrielle: développements récents», p. 173-192.

Miles, M.B. et A.M. Huberman (1991), Analyse des données qualitatives: recueil de nouvelles méthodes, Bruxelles, De Boeck Université.

ORGANISATION DE COOPÉRATION ET DE DÉvELOPPEMENT ÉCONOMIQUES - OCDE (1997), Manuel d'Oslo-Principes directeurs proposés pour le recueil et l'interprétation des données sur l'innovation technologique, Paris, Les éditions de l'OCDE/Eurostat.

PAturel, R. et N. BARriol (1999), «Les PME et la qualité: diffuser l'innovation en gestion grâce à une démarche collective», Revue française de gestion, ${ }^{\circ} 122$, janvier-février, p. 61-70.

ReIx, R. (1995), «Savoir tacite et savoir formalisé dans l'entreprise», Revue française de gestion, septembre-octobre, p. 17-28.

RoBERTSON, P.L. et R.N. LANGLOIS (1995), «Innovation, networks, and vertical integration», Research Policy, vol. 24, n 4, juin, p. 543-562.

Rogers, E. (1995), Diffusion of Innovations, $4^{\mathrm{e}}$ édition, New York, The Free Press.

Rosenkopf, L. et M.L. Tushman (1994), «The coevolution of technology and organization», dans A.C. Baum et J.V. Singh (dir.), Evolutionary Dynamics of Organizations, New York, Oxford University Press, p. 403-424.

Rothwell, R. (1978), «Small and medium sized manufacturing firms and technological innovation», Management Decision, vol. 16, $\mathrm{n}^{\circ}$ 6, p. 362-370.

TeECE, D.J. (1988), «Technical change and the nature of the firm», dans G. Dosi et al. (dir.), Technical Change and Economic Theory, Londres, Pinter Publishers, 646 p.

VAN DE VEN, A.H. (1986), «Central problems in the management of innovation», Management Science, vol. 32, n 5 , mai, p. 590-607.

Revue internationale P.M.E., vol. 19, nº 1, 2006

(C) 2006 - Presses de l'Université du Québec

Édifice Le Delta I, 2875, boul. Laurier, bureau 450, Québec, Québec G1V 2M2 • Tél.: (418) 657-4399 - www.puq.ca

Tiré de: Revue internationale P.M.E., vol. 19, no 1, sous la direction de Louis Raymond • PME1901N

Tous droits de reproduction, de traduction et d'adaptation réservés 
VAN DE VEN, A.H. et R. Garud (1994), «The coevolution of technical and institutional events in the development of an innovation», dans A.C. Baum et J.V. Singh (dir.), Evolutionary Dynamics of Organizations, New York, Oxford University Press, p. $425-443$.

VON HIPPEL, E. (1978), «Successful industrial products from customer ideas», Journal of Marketing, vol. 42, $\mathrm{n}^{\circ}$ 1, p. 39-49.

YIN, R.L. (1994), Case Study Research, Design and Methods, $2^{\mathrm{e}}$ édition, Thousand Oaks, Cal., Sage Publications, Applied Social Research Methods Series, vol. 5.

Revue internationale P.M.E., vol. 19, nº 1, 2006

(C) 2006 - Presses de l'Université du Québec

Édifice Le Delta I, 2875, boul. Laurier, bureau 450, Québec, Québec G1V 2M2 • Tél.: (418) 657-4399 - www.puq.ca

Tiré de: Revue internationale P.M.E., vol. 19, no 1, sous la direction de Louis Raymond • PME1901N

Tous droits de reproduction, de traduction et d'adaptation réservés 